\title{
Acoustic wave propagation in a circular cosh duct carrying a mean flow
}

\author{
Charles Thompson \\ Department of Electrical Engineering, University of Lowell, One University Avenue, Lowell, Massachusetts \\ 01854
}

\author{
Rahul Sen \\ Department of Engineering Science and Mechanics, Virginia Polytechnic Institute and State University, \\ Blacksburg, Virginia 24061
}

(Received 10 March 1987; accepted for publication 13 April 1987)

\begin{abstract}
An analysis of acoustic wave propagation in a waveguide carrying an incompressible mean flow is presented. The radius of the waveguide is taken to vary slowly as a function of axial location. It is shown that the dynamic behavior of the enclosed fluid can be parametrized by the small parameter $\epsilon$, where $\epsilon$ is the ratio of the typical duct radius $R_{0}$ and the wall wavelength $L_{0}$. An analytical solution for the pressure field in the duct is given in terms of a regular perturbation expansion in $\epsilon$. The method of matched asymptotic expansions is used to evaluate the refractive effect of a thin mean-flow boundary layer on the acoustic pressure field. It is shown that in the case where the duct geometry conforms to that of a circular cosh duct the effect of higher-order turning points in the wave equation can be effectively handled by a closed-form solution that approximately solves the governing equations. The results of analysis are compared to those obtained using numerical methods.
\end{abstract}

PACS numbers: 43.20.Mv

\section{INTRODUCTION}

Many aspects of acoustic wave propagation in ducts carrying a mean flow have been studied over the past 20 years. One reason for the prolonged interest in such problems is that flow ducts represent the quintessential element in processing systems, aircraft engines, and combustion systems. In general, the duct may have a cross section that varies along its axis as well as spatial variations in the mean velocity distribution. Hence, the problem precludes exact analytical solution. As a result, a number of investigators ${ }^{1-6}$ have resorted to approximate analytical techniques as well as numerical methods to determine the problem solution. However, two issues have not been resolved satisfactorily. The first deals with the refractive effect of a thin mean-flow boundary layer in cases where the acoustic wavenumber is not a constant as a function of the axial position. The second is the effect of a higher-order turning point resulting from changes in the acoustic wavelength as the pressure disturbance propagates along the duct.

The wave-envelope method (WEM) has recently been used to solve similar duct acoustics problems. ${ }^{1,2}$ However, WEM is essentially a lowest-order implementation of WKB theory. Hence, it yields solutions that are valid only outside the vicinity of turning points. As the axial wavenumber goes through zero, it is well known that a higher-order approximation is necessary if a uniformly valid solution for the pressure is to be obtained.

In this article we demonstrate that direct use of a regular perturbation sequence for the axial variation of pressure is free of such serious drawbacks. The turning-point features are fully contained in the zeroth-order problem. As a result, the perturbation series is automatically uniformly valid in the axial direction.
The specific problem addressed in this article is acoustic wave propagation in a waveguide carrying a mean flow. We will obtain an approximate solution for the pressure field using a regular perturbation sequence in $\epsilon$. Shearing of the mean flow is taken to be confined to a thin layer of $O(\Delta)$, where $\Delta \ll R_{0}$, the typical wall radius. As a result of the rapid radial variation of the mean-flow velocity in the vicinity of the walls, the radial variation of the pressure will be analyzed using the method of matched asymptotic expansions.

The enclosed fluid is assumed to be inviscid, isentropic and to respond linearly to harmonic excitation. In our analysis, we examine wave propagation in waveguides of circular cross section that have a radius that varies as a function of the horizontal position $X$ (see Fig. 1.). The degree of variation in duct geometry is measured by a small parameter $\epsilon$, where $\epsilon$ is the ratio of the two length scales $R_{0}$ and $L_{0}$. Here, $R_{0}$ is a measure of the typical duct radius and $L_{0}$ represents the typical wall wavelength. Throughout our analysis we will assume that the wall slope is $O(\epsilon)$ :

$$
\frac{d R(X)}{d X}=O\left(\frac{R_{0}}{L_{0}}\right)=O(\epsilon) .
$$

Section I will be devoted to the nondimensionalization of the linear inviscid equations of motion. The pressure and particle velocities will be expressed in terms of a truncated asymptotic sequence in $\epsilon$. In Secs. II and III we will present the solution for the coefficients of this asymptotic sequence. It will be shown that a closed-form approximate solution for the pressure can be obtained if the waveguide geometry takes the form of a circular cosh duct. An analysis of the pressure in the region of the mean-flow boundary layer is also given. The mean-flow boundary layer is shown to give rise to a modulation of the acoustic pressure amplitude. Section IV will be devoted to a discussion of the results of our analysis. 


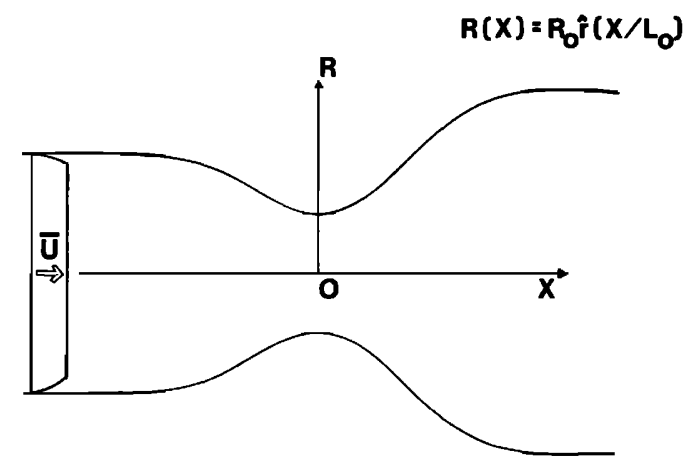

FIG. 1. Schematic of a circular cosh duct.

\section{NONDIMENSIONALIZATION OF THE EQUATIONS OF MOTION}

In this section we will present the equations governing inviscid linear wave propagation in a waveguide carrying an incompressible mean flow. We will also present a scheme for nondimensionalizing the equations of motion. The resulting nondimensional equations will govern acoustic wave propagation in the duct.

The oscillatory particle velocities $U^{*}, V^{*}, W^{*}$ and the pressure $P^{*}$ in the duct satisfy the following equations:

$\rho_{0} \frac{\partial U^{*}}{\partial T}+\rho\left(\frac{\partial\left(U^{*} \bar{U}\right)}{\partial X}+\bar{V} \frac{\partial U^{*}}{\partial R}+V^{*} \frac{\partial \bar{U}}{\partial R}\right)+\frac{\partial P^{*}}{\partial X}=0$,

$\rho_{0} \frac{\partial V^{*}}{\partial T}+\rho\left(\frac{\bar{U} \partial V^{*}}{\partial X}+U^{*} \frac{\partial \bar{V}}{\partial X}+\frac{\partial\left(V^{*} \bar{V}\right)}{\partial R}\right)+\frac{\partial P^{*}}{\partial R}=0$,

$\rho_{0} \frac{\partial W^{*}}{\partial T}+\rho\left[\frac{\bar{U} \partial W^{*}}{\partial X}+\bar{V}\left(\frac{\partial W^{*}}{\partial R}+\frac{W^{*}}{R}\right)\right]+\frac{1}{R} \frac{\partial P^{*}}{\partial \Theta}=0$,

$\frac{1}{c_{0}^{2}} \frac{\partial P^{*}}{\partial T}+\rho_{0}\left(\frac{\partial U^{*}}{\partial X}+\frac{1}{R} \frac{\partial\left(R V^{*}\right)}{\partial R}+\frac{1}{R} \frac{\partial W^{*}}{\partial \Theta}\right)$

$+\frac{1}{c_{0}^{2}}\left(\bar{U} \frac{\partial P^{*}}{\partial X}+\bar{V} \frac{\partial P^{*}}{\partial R}\right)=0$,

where $P^{*}$ is the oscillatory pressure, $\rho_{0}$ is the rest density of the fluid, $c_{0}$ is the speed of sound, and $U^{*}, V^{*}$ and $W^{*}$ represent the oscillatory particle velocities in the $X, R$, and $\theta$ directions, respectively.

Consider a rigid-walled waveguide of circular cross section with the radius varying slowly along the $x$ axis (Fig. 1). If the fluid in the duct behaves in an inviscid fashion under acoustic excitation, the only boundary condition that must be satisfied is the no-penetration condition on the boundary. This may be written as

$$
V^{*}=R_{0} \frac{d \hat{r}\left(X / L_{0}\right)}{d X} U^{*}
$$

at $R=R_{0} \hat{r}\left(X / L_{0}\right)$.

The dynamic behavior of the enclosed fluid is character- ized by the relationship between two length scales, namely, (1) the typical duct radius $R_{0}$ and (2) the typical wall wavelength $L_{0}$. We will assume $R(X)$ is $O\left(R_{0}\right)$. The slope of the duct is assumed to be $O(\epsilon)$. Hence,

$$
R_{0} \frac{d \hat{r}\left(X / L_{0}\right)}{d\left(X / L_{0}\right)}=\epsilon \hat{r}^{\prime}\left(\frac{X}{L_{0}}\right)=O(\epsilon),
$$

where $\epsilon=R_{0} / L_{0}$ and $\hat{r}^{\prime}=O(1)$.

The normal component of the mean velocity is zero on the wall. Given that wall slope is $O(\epsilon)$, we expect the typical amplitude of the vertical component of the mean velocity $\bar{V}_{0}$ to be related to the typical amplitude of the horizontal velocity $\bar{U}_{0}$ as

$$
\bar{V}_{0}=\epsilon \bar{U}_{0} .
$$

The variation of the mean velocity with $X$ is related to the magnitude of the wall wavelength which is $O\left(L_{0}\right)$. For the acoustic quantities, the spatial variations are related to the acoustic wavelength $\lambda=O\left(R_{0}\right)$. Hence, the mean-flow velocities will be functions of a slow nondimensional axial variable $x_{1}$, where

$$
x_{1}=\epsilon\left(X / R_{0}\right) .
$$

Along the radial direction the mean-flow velocities vary rapidly as $R-R_{0} \hat{r}\left(X / L_{0}\right)$ approaches $\Delta$, where $\Delta$ is the boundary-layer thickness of $\bar{U}$ and $\bar{V}$. Therefore, the mean-flow variables will be functions of a fast radial variable $(r-\hat{r}) / \delta$, where

$$
\frac{(r-\hat{r})}{\delta}=\frac{R-R_{0} \hat{r}}{\Delta}=\left(\frac{R-R_{0} \hat{r}}{R_{0}}\right) \frac{1}{\delta}
$$

and

$$
\delta=\Delta / R_{0} .
$$

The consequences of this choice of scaling will be addressed shortly. Our nondimensionalization scheme for the oscillatory variables is

$$
u=U^{*} / U_{0}, \quad v=V^{*} / U_{0}, \quad w=W^{*} / U_{0},
$$

and

$$
P=P^{*} /\left(\omega \rho_{0} U_{0} R_{0}\right)
$$

For the coordinates the nondimensionalizations are

$$
x=x / R_{0}, \quad x_{1}=x / L_{0}=x \epsilon, \quad r=R / R_{0},
$$

where

$$
\begin{aligned}
& \bar{u}\left(x_{1}, \frac{r-\hat{r}}{\delta}\right)=\frac{\bar{U}}{\bar{U}_{0}}, \quad \bar{v}\left(x_{1}, \frac{r-\hat{r}}{\delta}\right)=\frac{\bar{V}}{\bar{V}_{0}}=\frac{\bar{V}}{\left(\epsilon \bar{U}_{0}\right)}, \\
& t=\frac{T}{\left(R_{0} / c_{0}\right)}, \quad k=\frac{\omega}{c} R_{0}, \quad \text { and } m=\frac{\bar{U}_{0}}{c_{0}} .
\end{aligned}
$$

Choosing a time dependence of the form $e^{-i k t}$ and a $\theta$ dependence $e^{i n \theta}$, Eqs. (1) in terms of the nondimensional variables are

$$
\begin{aligned}
& -i k u+m\left(u_{x} \bar{u}+v \bar{u}_{r}\right)+\epsilon m\left(u_{x_{1}} \bar{u}+u \bar{u}_{x_{1}}+\bar{v} u_{r}\right)+p_{x}+\epsilon p_{x_{1}}=0, \\
& -i k v+m\left(\bar{u} v_{x}\right)+\epsilon m\left[(\bar{v} v)_{r}+\epsilon u \bar{v}_{x_{1}}\right]+p_{r}=0, \\
& -i k w+m\left(\bar{u} w_{x}\right)+\epsilon m\left[\bar{v} w_{r}+\bar{v}(w / r)+\bar{u} w_{x_{1}}\right]+(i n / r) p=0, \\
& -i k p+\left[u_{x}+(1 / r)(r v)_{r}+(i n / r) w\right]+m \bar{u} p_{x}+\epsilon u_{x_{1}}+m \epsilon\left(\bar{u} p_{x_{1}}+\bar{v} p_{r}\right)=0,
\end{aligned}
$$


for the complex amplitudes, and the steady continuity equation becomes

$$
\bar{u}_{x_{1}}+(1 / r)(r \bar{v})_{r}=0 .
$$

We point out that the influence of the mean vertical velocity $\bar{v}$ on the acoustic wave is measured by the parameter $\epsilon$, which is the wall slope. The wall boundary condition is

$$
v\left(x_{1}, x, \hat{r}\right)=\epsilon \hat{r}^{\prime}\left(x_{1}\right) u\left(x_{1}, x, \hat{r}\right)
$$

at $r=\hat{r}\left(x_{1}\right)$. We will express the complex amplitudes $p, u, v$, and $w$ in terms of an asymptotic sequence in integer powers of $\epsilon$. Successive approximations for the pressure and particle velocity can be determined by evaluating higher-order coefficients of this sequence:

$$
(p, u, v, w)=E_{1}(p, u, v, w),
$$

where

$$
E_{n} f\left(x_{1}, x, r, \delta ; \epsilon\right)=\sum_{n=0}^{N} f_{n}\left(x_{1}, x, r, \delta\right) \epsilon^{n}+O\left(\epsilon^{N+1}\right) .
$$

\section{SOLUTION AT ZEROTH ORDER}

In this section we will consider the solution of the zeroth-order problem. Our primary concern at this order of approximation will be to determine how an acoustic wave propagates within and outside the mean-flow boundary layer. To this end the space within the waveguide will be split into two regions. The first will be called the outer region and the second will be termed the inner region. The outer region is defined as the region of space where $(r-\hat{r})$ is $O(1)$. This approach is merited as a result of the fact that both meanflow variables $\bar{u}$ and $\bar{v}$ are functions of the fast radial variable $(r-\hat{r}) / \delta$ and the slow axial variable $x_{1}$. In the outer region the shear contributed by the radial variation of the meanflow profile has little effect on acoustic wave propagation. The major influence of the mean flow is that of modifying the axial variation of the acoustic wavelength. The inner region is defined to be the region of space where $(r-\hat{r})$ is $O(\delta)$. This region is characterized by a rapid variation in the meanflow profile. The shear of the mean flow in this region results in refraction of the acoustic wave. As a result of the local validity of the pressures obtained, the solutions in each of the aforementioned regions will be singular in the other region. Therefore, to obtain a globally valid solution for the acoustic pressure we will use the method of matched asymptotic expansions. ${ }^{7}$ The equations governing acoustic propagation at this order of approximation are obtained by substituting Eq. (3) into Eqs. (2) and equating like terms in $\epsilon^{0}$ :

$$
\begin{aligned}
& -i k u_{0}+m\left(u_{0 x} \bar{u}+v_{0} \bar{u}_{r}\right)+p_{0 x}=0, \\
& -i k v_{0}+m\left(\bar{u} v_{0 x}\right)+p_{0 r}=0, \\
& -i k w_{0}+m\left(\bar{u} w_{0 x}\right)+(i n / r) p_{0}=0, \\
& -i k p_{0}+\left[u_{0 x}+(1 / r)\left(r v_{0}\right)_{r}\right. \\
& \left.\quad+(i n / r) w_{0}\right]+m\left(\bar{u} p_{0 x}\right)=0,
\end{aligned}
$$

where the pressure $p_{0}$ satisfies the wave equation

$$
\begin{aligned}
L\left(p_{0}, \bar{u}, \bar{v}, k\right)= & -k^{2} p_{0}-\left[1-(m \bar{u})^{2}\right] p_{0 x x} \\
& -(1 / r)\left(r p_{0 r}\right)_{r}+\left(n^{2} / r^{2}\right) p_{0} \\
& -2 m \bar{u} p_{0 x} i k-2 m\left(\bar{u}_{r} v_{0 x}\right)=0 .
\end{aligned}
$$

The boundary condition at this order is

$$
v_{0}=0 \text { at } r=\hat{r}\left(x_{1}\right) \text {. }
$$

Note that solving Eq. (8), as it stands, would yield a pressure field that is globally valid in the radial direction. This can only be achieved numerically. Recall, however, that $\bar{u}$ and $\bar{v}$ are functions of the fast radial variable $(r-\hat{r}) / \delta$. This implies that the acoustic pressure inside and outside the meanflow boundary layer can be evaluated in terms of locally valid solutions in each of the respective regions. These solutions will be in the form of singular asymptotic expansions in terms of gauge functions in $\delta$. A globally valid solution for the pressure can then be determined by matching the local solutions in the radial direction.

\section{A. Outer solution}

In the limit of $\delta$ approaching zero the contribution of the term $\left(\bar{u}_{r} v_{0 x}\right)$ in Eq. (8) is null. Therefore, in the outer region, the pressure satisfies the equation

$$
\begin{aligned}
& \left(1-m^{2} \bar{u}_{0}^{2}\right) p_{0 x x}+(1 / r)\left(r p_{0 r}\right)_{r} \\
& \quad+\left(k^{2}-n^{2} / r^{2}\right) p_{0}+2 i k m \bar{u}_{0} p_{0 x}=0,
\end{aligned}
$$

where

$$
\bar{u}_{0}=\bar{u}\left[(r-\hat{r}) / \delta=\infty, x_{1}\right] .
$$

The solution for the pressure can be expressed as

$p_{0}\left(r, x, x_{1}\right)=f\left(r, x_{1}\right)\left[\exp \left(i k \int_{-\infty}^{x} \frac{m \bar{u}_{0}}{1-m^{2} \bar{u}_{0}^{2}} d x\right) g\left(x, x_{1}\right)\right]$.

Substituting Eq. (11) into Eq. (10) yields for $g\left(x, x_{1}\right)$

$$
g_{x x}+\left(\hat{k}^{2}-\hat{\gamma}^{2} / \hat{r}^{2}\right) g=0
$$

and for $f\left(r, x_{1}\right)$

$$
f_{r r}+1 / r f_{r}+\left(\gamma^{2} / \hat{r}^{2}-n^{2} / r^{2}\right) f=0,
$$

where

$$
\begin{aligned}
& \hat{\gamma}^{2}=\gamma^{2} /\left[1-\left(m \bar{u}_{0}\right)^{2}\right], \\
& \hat{k}^{2}=k^{2} /\left[1-\left(m \bar{u}_{0}\right)^{2}\right]^{2},
\end{aligned}
$$

and

$$
\bar{u}_{0}=\bar{u}\left[x_{1},(r-\hat{r}) / \delta=\infty\right] .
$$

The value of $\gamma$ must be such that the boundary condition $v_{0}=0$ at $r=\hat{r}$ is satisfied by the inner solution, which is dependent on the influence of the mean-flow boundary layer. Examining Eq. (12), we see that both $\hat{k}$ and $\hat{\gamma}$ are slowly varying functions of $x$. As $x$ approaches $\pm \infty$ the leadingorder behavior of the pressure is

$$
\begin{aligned}
p_{0} \rightarrow f\left(r, x_{1}\right) \exp \left(i k \int_{-\infty}^{x} \frac{m \bar{u}_{0}}{1-m^{2} \bar{u}^{2}} d x\right) \\
\times \exp \left[ \pm i \int_{-\infty}^{x}\left(\hat{k}^{2}-\frac{\hat{\gamma}^{2}}{\hat{r}^{2}}\right)^{1 / 2} d x\right],
\end{aligned}
$$

which is identical to the result obtained via the wave-envelope method. ${ }^{1,2}$ However, if $\left(\hat{k}^{2}-\hat{\gamma}^{2} / \hat{r}^{2}\right)$ approaches zero in any interval in $x$, the aforementioned result is not uniformly valid in $x$. This state of affairs is a result of the assumption that $\left(\hat{k}^{2}-\hat{\gamma}^{2} / \hat{r}^{2}\right)$ is $O(1)$ for all $x$. The underlying assump- 
tion in the wave-envelope method is that

$$
\left|\int \sqrt{\left(\hat{k}^{2}-\frac{\hat{\gamma}^{2}}{\hat{r}^{2}}\right)} d x\right|>\frac{1}{4}\left|\ln \left(\hat{k}^{2}-\frac{\hat{\gamma}^{2}}{\hat{r}^{2}}\right)\right|
$$

for all $x$. As the acoustic pressure wave approaches a cutoff region, this condition is not satisfied. Furthermore, the wave-envelope method is simply a zeroth-order implementation of WKB theory. If we assume that $\hat{k}$ and $\hat{\gamma}$ are slowly varying in $x$ and relax this assumption on $\hat{r}$, we can retain the behavior of the pressure through regions where axial wavenumber becomes small, or even negative, and as a result we can obtain a globally valid approximation to the $x$ dependence of pressure. The utility of such an approach can be readily exemplified in the case where the axial variation in the radius takes the form of a "circular-cosh" duct. If

$$
\begin{aligned}
\left(\hat{r}^{-} / \hat{r}\right)^{2}= & \beta^{2}-\left(\beta^{2}-1\right) e^{2 \mu} \cosh (2 \mu) \\
& +\left(\beta^{2}-1\right) e^{2 \mu}\left[\cosh ^{2}(\mu) \operatorname{sech}^{2}(\epsilon x-\mu)\right. \\
& -\sinh (2 \mu) \tanh (\epsilon x-\mu)],
\end{aligned}
$$

where

$$
\begin{aligned}
& \tau=\hat{r}(\infty) / \hat{r}(-\infty)=\hat{r}^{+} / \hat{r}^{-}, \\
& \beta=\hat{r}(-\infty) / \hat{r}(0)=\hat{r}^{-} / \hat{r}_{0},
\end{aligned}
$$

and

$$
\mu=\frac{1}{4} \ln \left[\left(\beta^{2}-1 / \tau^{2}\right) /\left(\beta^{2}-1\right)\right] .
$$

It can be shown that $g\left(x, x_{1}\right)$ is

$$
\begin{aligned}
g\left(x, x_{1}\right)= & e^{i / 2\left(k^{+}-k^{-}\right)(x-\mu / \epsilon)}\left(e^{(x \epsilon-\mu)}+e^{-(x \epsilon-\mu)}\right)^{\Psi} \\
& \times\left[A_{0} F(a, b ; c ; z)+B_{0} z^{1-c}\right. \\
& \times F(b-c+1, a-c+1 ; 2-c ; z)],
\end{aligned}
$$

where

$$
\begin{aligned}
& \gamma^{-}=\lim _{x \rightarrow-\infty} \hat{\gamma}, \\
& k^{+}=\lim _{x \rightarrow \infty}\left(\hat{k}^{2}-\hat{\gamma}^{2} / \hat{r}^{2}\right)^{1 / 2}, \\
& k^{-}=\lim _{x \rightarrow-\infty}\left(\hat{k}^{2}-\hat{\gamma}^{2} / \hat{r}^{2}\right)^{1 / 2}, \\
& a=\frac{1}{2}\left[1-i\left(k^{+} / \epsilon+k-/ \epsilon-s\right)\right], \\
& b=\frac{1}{2}\left[1-i\left(k^{+} / \epsilon+k^{-} / \epsilon+s\right)\right], \\
& c=1-i k^{+} / \epsilon, \\
& \Psi=\frac{1}{2}\left(k^{+}+k^{-}\right) / \epsilon, \\
& z=\left(e^{2(\epsilon x-\mu)}+1\right)^{-1}, \\
& s=\left\{4 e^{2 \mu}\left(\beta^{2}-1\right)\left[\gamma^{-2} /\left(\epsilon \hat{r}^{-}\right)^{2}\right] \cosh ^{2}(\mu)-1\right\}^{1 / 2}, \\
& \mu=\frac{1}{4} \ln \left[\left(\beta^{2}-1 / \tau^{2}\right) /\left(\beta^{2}-1\right)\right],
\end{aligned}
$$

and $F(a, b ; c ; z), F(b-c+1, a-c+1 ; 2-c ; z)$ are hypergeometric functions. Here, $A_{0}$ and $B_{0}$ are arbitrary at this point. Insofar as $\hat{\gamma}$ and $\hat{k}$ are slowly varying in $x$, Eq. (14) represents an exact solution of Eq. (12). As $x$ approaches $\pm \infty$, the leading behavior of the pressure must be identical to that obtained using the wave-envelope method and regular through any turning points in $x$. Hence, $B_{0}$ must be equal to zero. In the limit of $\delta$ approaching zero the boundary condition on $f$ becomes $f_{r}=0$ on the walls of the duct. To determine the influence of the mean-flow boundary layer we must examine the inner region. Using the result given in Eq. (14) and solving Eq. (13) subject to the boundary condition $f_{r}=0$ at $(r=\hat{r}+O(\delta)$ yield the following results for the acoustic pressure:

$$
\begin{aligned}
& \text { for } x \leqslant 0, \\
& \qquad \begin{aligned}
E_{0} p= & C_{l, n}\left(\dot{x}_{1}\right) \exp [i(n \theta-k t)] \exp \left(i k \int_{-\infty}^{x} \frac{m \bar{u}_{0}}{1-m^{2} \bar{u}_{0}^{2}} d x\right) J_{n}\left(\frac{\gamma_{l, n} r}{\hat{r}}\right) \\
& \times\left\{\exp (i k-x)\left[\phi_{+}^{\left(k^{+}-k^{-}\right) / \epsilon} F(c-a, c-b ; c-a-b+1 ; 1-z)\right]\right\} \\
& +R \exp (-i k-x)\left[\phi_{-}^{\left(k^{+}+k^{-}\right) / \epsilon} F(a, b ; a+b-c+1 ; 1-z)\right]+O(\epsilon)+O(\delta),
\end{aligned}
\end{aligned}
$$

and for $x>0$

$$
\begin{aligned}
E_{0} p= & C_{l, n}\left(x_{1}\right) \exp (i n \theta-k t)\left[\exp \left(i k \int_{-\infty}^{x} \frac{m \bar{u}_{0}}{1-m^{2} \bar{u}_{0}^{2}} d x\right)\right] J_{n}\left(\frac{\gamma_{l, n} r}{\hat{r}}\right) \\
& \times T \exp (i k+x)\left[\phi_{-}^{i\left(k^{+}-k^{-}\right) / \epsilon} F(a, b ; c ; z)\right]+O(\epsilon)+O(\delta),
\end{aligned}
$$

where

$$
\begin{aligned}
& \phi_{-}=1+e^{2(x \epsilon-\mu)}, \\
& \phi_{+}=1+e^{-2(x \epsilon-\mu)}, \\
& R=\frac{\Gamma(b) \Gamma(a) \Gamma(c-a-b)}{\Gamma(a+b-c) \Gamma(c-a) \Gamma(c-b)} e^{i 2 k^{-\mu},}, \\
& T=\frac{\Gamma(b) \Gamma(a)}{\Gamma(c) \Gamma(c-a-b)} e^{i\left(k^{-}-k^{+}\right) \mu} .
\end{aligned}
$$

Here, $R$ and $T$ represent the complex reflection and transmission coefficients, respectively. As mentioned earlier, we must examine the pressure in the inner region to find the appropriate boundary condition on $f$.

\section{B. Inner solution}

Now let us direct our attention to the oscillatory motion of the fluid in proximity to the walls of the duct. As $(r-\hat{r})$ becomes $O(\delta)$, the term $\left(\bar{u}_{r} v_{0 x}\right)$ in Eq. (8) becomes $O(1)$. The increase in the magnitude of this term is the result of the rapid deceleration of the mean flow near the walls. As a result, the axial wavenumber varies rapidly as a function of the radial position. Therefore, the equations described in the 
previous section are singular in the limit of $(r-\hat{r})$ approaching $\delta$. Hence, a locally valid set of equations must be derived to describe the fluid motion in this region of space. When $(r-\hat{r})$ becomes $O(\delta), \delta v_{0}$ becomes of $O(1)$. Using this information, we can derive a set of local variables and equations governing the motion of the fluid. The inner variables are

$$
\begin{aligned}
& r^{*}=(r-\hat{r}) / \delta, \quad x=x^{*}, \quad u^{*}=u, \quad \bar{u}^{*}=\bar{u}, \\
& v^{*}=v / \delta-\hat{r}^{\prime} \epsilon u^{*}, \quad \bar{v}^{*}=\bar{v} / \delta-\hat{r}^{\prime} \bar{u} .
\end{aligned}
$$

Substituting the aforementioned variables into Eqs. (2) and truncating the resulting equations to terms of $O(1)$, the following equation is obtained for the zeroth-order pressure:

$$
\begin{aligned}
p_{r^{*}, *}^{*} & +\frac{\delta}{\delta r^{*}+\hat{r}} p_{r^{*}}^{*}-\frac{2 m \bar{u}_{r^{*}}}{\hat{I}} \int^{x} \frac{p_{r^{*} x}^{*}}{m \bar{u}^{*}} \hat{I} \\
& +\delta^{2}\left[\left(k^{2}-\frac{n^{2}}{\left(\delta r^{*}+\hat{r}\right)^{2}}\right) p^{*}+\left(1-m^{2} \bar{u}^{2}\right)\right. \\
& \left.\times p_{x^{*} x^{*}}^{*}+i 2 k m \bar{u}^{*} p_{x^{*}}^{*}\right]=O(\epsilon),
\end{aligned}
$$

where

$$
\hat{I}=\exp \left(-i k \int^{x} \frac{d x}{m \bar{u}^{*}}\right)
$$

The boundary condition is that

$$
p_{r^{*}}=0 \text { at } r^{*}=0 \text {. }
$$

The inner pressure and the eigenvalue are sought in terms of asymptotic expansions in $\delta$ :

$$
\begin{aligned}
& p^{*}\left(x, x_{1}, r^{*}, \gamma_{l, n} / \hat{r}\right)= p^{0 *}\left(x, x_{1}, r^{*} \gamma_{0 l, n} / \hat{r}\right) \\
&+\delta^{j} p^{j *}\left(x, x_{1}, r^{*} \gamma_{l, n} / \hat{r}\right)+o\left(\delta^{j}\right) \\
& \gamma_{l, n}=\gamma_{0 l, n}+\delta_{\gamma 1 l, n}+o(\delta),
\end{aligned}
$$

where $j$ must be determined from matching the inner and outer solutions.

As $\delta$ approaches zero the outer limit of $p^{*}$ must be equal to the inner limit of $p_{0}$. The pressure in the inner region must also satisfy the aforementioned boundary condition. Therefore, the zeroth-order approximation for the local pressure is

$$
p^{0 *}=p_{0}(x, \hat{r}) .
$$

This, of course, is just a statement of continuity in pressure. For the boundary condition to be satisfied, the zeroth-order approximation to the eigenvalue is

$$
\gamma_{l, n}=\gamma_{0 l, n}+O(\delta),
$$

where $\gamma_{0 l, n}$ is identically equal to the eigenvalue evaluated in the absence of shear in the mean flow. The influence of shear on both the pressure and eigenvalue must be determined at higher order.

The next term in the asymptotic sequence for the local pressure is of $O\left(\delta^{j}\right)$, where $j$ must be determined through matching conditions imposed by the outer pressure field. The pressure at $O\left(\delta^{j}\right)$ satisfies the equation

$$
\begin{gathered}
p_{r *, *}^{j *}-\frac{2 \bar{u}_{r^{*}}}{\hat{I} \bar{u}} \int^{x} p_{r_{x}^{*}}^{j *} \hat{I}+\delta^{2-j}\left[\left(k^{2}-n^{2} / \hat{r}^{2}\right) p^{0 *}\right. \\
\left.+\left(1-m^{2} \bar{u}^{2}\right) p_{x^{*} x^{*}}^{0 *}+i 2 k m \bar{u}^{*} p_{x^{*}}^{0 *}\right]=0 .
\end{gathered}
$$

In order to solve this equation we utilize spatial Fourier transforms. Taking the spatial Fourier transform in $x$ of the pressure in Eq. (20) yields

$$
p_{r_{r *}^{*}}^{j *}-\left(\frac{2 \bar{u}_{r^{*}} m i \hat{\omega}}{i \hat{\omega} \bar{u}-i k}\right) P_{r *}^{j *}+\widehat{A} \delta^{2-j}=0
$$

where

$$
\begin{aligned}
\hat{A}\left(\hat{\omega}, r^{*}\right)= & {\left[\left(\gamma_{l, n}^{2}-n^{2}\right) / \hat{r}^{2}\right] P^{0 *}-m^{2}\left(\bar{u}^{2}-\bar{u}_{0}^{2}\right) } \\
& \times P^{0 *}(i \widehat{\omega})^{2}+i 2 k m\left(\bar{u}-\bar{u}_{0}\right) P^{0 *}(i \widehat{\omega})
\end{aligned}
$$

and

$$
P^{j *}=\frac{1}{2 \pi} \int_{-\infty}^{\infty} P^{j *} e^{i \hat{\omega} x} d \widehat{\omega} .
$$

The solution for the $\delta^{j}$-order pressure coefficient is

$$
\begin{aligned}
P^{j *}= & B_{1}+B_{2} \int_{1}^{r^{*}}(i \hat{\omega} \bar{u} m-i k)^{2} d s-\delta^{2-j} \\
& \times \int_{1}^{r^{*}}(i \hat{\omega} \bar{u} m-i k)^{2} d s \int_{1}^{s} \frac{\hat{A} d s_{2}}{i \hat{\omega} \bar{u} m-i k)^{2}} .
\end{aligned}
$$

If $P_{r}^{j *}=0$ at $r^{*}=0$

$$
B_{2}=-\int_{0}^{1} \frac{\hat{A}}{(i \hat{\omega} \bar{u} m-i k)^{2}} .
$$

The coefficient $B_{1}$ and the gauge function $\delta^{j}$ must be determined through the matching of the inner and outer solutions in the radial direction. We will now direct our attention to the matching condition on the inner and outer solutions. Recall from the previous section that the outer pressure field is of the form $p_{0}\left(x, x_{1}, r \gamma_{l, n} / \hat{r}\right)$. Taking the spatial Fourier transform of the outer solution in $x$ yields $P_{0}\left(i \widehat{\omega}, x_{1}, r \gamma_{l, n} / \hat{r}\right)$. In the limit of $\gamma_{l, n}$ and $r$ approaching $\gamma_{0 l, n}$ and $\hat{r}$, respectively,

$$
\begin{aligned}
P_{0}\left(i \hat{\omega}, r \gamma_{l, n} / \hat{r}\right)= & P_{0}\left(i \hat{\omega}, \gamma_{0 l, n}\right) \\
& +\left.\left[\left(\gamma_{l, n}-\gamma_{0 l, n}\right)+(r-\hat{r})\right] P_{0 r}\right|_{\hat{r}, \gamma_{l l, n}} \\
& +\left[\left(\gamma_{l, n}-\gamma_{0 l, n}\right)^{2}+2\left(\gamma_{l, n}-\gamma_{0 l, n}\right)(r-\hat{r})\right. \\
& \left.+(r-\hat{r})^{2}\right]\left.\left(P_{0 r r} / 2 !\right)\right|_{\hat{r}, \gamma_{l l, n}}+\text { h.o.t. }
\end{aligned}
$$

Transforming this result into inner variables and truncating at order $\delta^{2}$ yield

$$
\begin{aligned}
P_{0}\left(\hat{\omega}, r \gamma_{l, n} / \hat{r}\right)= & P_{0}\left(i \widehat{\omega}, \gamma_{0 l, n}\right)+\left.\delta\left(\gamma_{1 l, n}+r^{*}\right) P_{0 r}\right|_{\hat{r}, \gamma_{0 l, n}} \\
& +\left.\delta^{2}\left(\gamma_{1 l, n}^{2}+2 \gamma_{1 l, n} r^{*}+r^{* 2}\right) \frac{P_{0 r r}}{2 !}\right|_{\hat{r}, \gamma_{0 l, n}} \\
& +o\left(\delta^{2}\right) .
\end{aligned}
$$

It is important to note that $P_{0 r}$ is zero in the aforementioned limit. Thus for the inner and outer pressures to match, the gauge function for the $P^{j *}$ coefficient must be $\delta^{2}$. Any other choice would result in an inconsistency at this order of the expansion. Matching the inner to the outer solution for the pressure we find

$$
\lim _{r_{* \rightarrow \infty}} P_{0}^{j *}=\left.\left(r^{*}+\gamma_{1 l, n}\right)^{2} \frac{P_{0 r r}}{2 !}\right|_{\hat{r}, \gamma_{0, n}} .
$$

The aforementioned result appears to differ from that obtained by Myers and Chuang. ${ }^{6}$ In their investigation they left the eigenvalue as a free parameter in the expansion. This choice resulted in transcendental equation of the eigenvalue 
involving the parameter $\delta$. As $\delta$ approaches zero the eigenvalue can be represented by an asymptotic sequence in $\delta$. In such a case, their results would be in agreement with those presented here. The second-order coefficient of the local pressure is

$$
\begin{aligned}
P^{j *}= & \int_{0}^{1} \frac{\hat{A}}{(\hat{\omega} \bar{u} m-i k)^{2}} d r^{*} \\
& \times\left(-\int_{1}^{r^{*}}(i \hat{\omega} \bar{u} m-i k)^{2} d r^{*}+\left(i \hat{\omega} \bar{u}_{0} m-i k\right)^{2}\right) \\
& -\int_{1}^{r^{*}}(\hat{\omega} \bar{u} m-i k)^{2} d s \int_{1}^{s} \frac{\hat{A}(\hat{\omega}, \tau) d \tau}{(i \hat{\omega} \bar{u} m-i k)^{2}} \\
& -\left(\gamma_{1 l, n}^{2}-1\right) \hat{A} /\left.2\right|_{r^{*}=1} .
\end{aligned}
$$

The eigenvalue valid to second order in $\delta$ is

$$
\gamma_{l, n}=\gamma_{0 l, n}+\delta^{1} \gamma_{1 l, n}+O\left(\delta^{2}\right),
$$

where

$$
\gamma_{1, n}=\int_{0}^{1}\left(\frac{\hat{A}}{(i \hat{\omega} \bar{u} m-i k)^{2}}\right) \frac{\left.i \widehat{\omega} \bar{u}_{0} m-i k\right)^{2}}{\left.\hat{A}\right|_{, *=1}}-1 .
$$

Examining this result for $\gamma_{l, n}$ we see that shear in the mean flow serves to modify the eigenvalue as a function of the spatial frequency. Hence, we expect the eigenvalue to vary with axial position. The degree of modification is dependent on the spatial bandwidth of the outer pressure field. Hence, the outer solution given in Eqs. (15) and (16) is valid to $O(\delta)$ if $\gamma_{l, n}$ is taken to be a constant in $x$. It is also important to note that the result is singular as $\gamma_{0 l, n}$ becomes $O(1 / \delta)$. In such a case the refractive effect of the mean-flow boundary layer would not be confined to the inner region.

\section{Composite solution}

Both inner and outer solutions given in the previous two sections can now be combined into a single composite solution. This solution is uniformly valid throughout the domain of interest. This composite expansion is

$$
E_{0,2} p=p_{0}+\frac{\delta^{2}}{2 \pi} \int_{-\infty}^{\infty}\left[P^{j *}\left(i \widehat{\omega}, r^{*}\right)-P^{j *}(i \widehat{\omega}, 1)\right] e^{i \hat{\omega} x} d \widehat{\omega},
$$

where the expressions for $p_{0}$ are given in Eqs. (15) and (16). The solution given above is valid to $O(\delta)$ in the outer region and valid to $O\left(\delta^{3}\right)$ in the inner region.

\section{SOLUTION AT FIRST ORDER}

In this section we will outline the solution of the firstorder problem. We will show that the function $C_{l, n}\left(x_{1}\right)$ given in Eqs. (15) and (16) satisfies a first-order differential equation in the slow variable $x_{1}$ and that this equation represents a solvability condition for our asymptotic representation of the pressure.

At order $\epsilon$ the operation governing the complex amplitude of the pressure $p_{1}$ is given in Eqs. (24)-(28).

$$
\begin{aligned}
& -i k u_{1}+m\left(u_{1 x} \bar{u}+v_{1} \bar{u}_{r}\right)+p_{1 x} \\
& \quad=-m\left(u_{0 x} \bar{u}+u_{0} \bar{u}_{x_{1}}+\bar{v} u_{0 r}\right) p_{0 x_{1}}, \\
& -i k v_{1}+m\left(\bar{u} v_{1 x}\right)+p_{1 r}=-m\left[\left(\bar{v} v_{0}\right)_{r}+\bar{u} v_{0 x_{1}}\right],
\end{aligned}
$$

$$
\begin{aligned}
& -i k w_{1}+m\left(\bar{u} w_{1 x}\right)+(i n / r) p_{1} \\
& \quad=-m\left[\bar{v} w_{0 r}+\bar{v}\left(w_{0} / r\right)+\bar{u} w_{0 x_{1}}\right] \\
& -i k p_{1}+\left[u_{1 x}+(1 / r)\left(r v_{1}\right)_{r}+(i n / r) w_{1}\right]+m \bar{u} p_{1 x} \\
& \quad=-u_{x_{1}}-m\left(\bar{u} p_{0 x_{1}}+\bar{v} p_{0 r}\right)
\end{aligned}
$$

where the pressure $p_{1}$ satisfies

$$
\begin{aligned}
L\left(p_{1}, \bar{u}, \bar{v}, k\right)= & -m\left(-i k+m \bar{u} \frac{\partial}{\partial x}\right)\left(\bar{u} p_{0 x_{1}}+\bar{v} p_{0 r}\right) \\
& -m\left(\bar{u} \frac{\partial}{\partial x_{1}}+\bar{v} \frac{\partial}{\partial r}\right)\left(-i k p_{0}+m \bar{u} p_{0 x}\right) \\
& -2 m\left[(\bar{v} / r)\left(w_{0} i n-u_{0 x}\right)+\bar{v}_{r}\right. \\
& \left.\times\left(v_{0 r}-u_{0 x}\right)+\bar{u}_{r} v_{0 x_{1}}\right]+2 p_{0 x x_{1}} \cdot(28)
\end{aligned}
$$

The boundary condition at this order of approximation is

$$
p_{1 r}=\hat{r}^{\prime}\left(x_{1}\right) p_{0 x} \text { at } r=\hat{r}\left(x_{1}\right) .
$$

Outside the boundary layer of the mean flow, Eq. (28) can be rewritten as

$$
\begin{aligned}
& \left(1-m^{2} \bar{u}_{0}^{2}\right) p_{1 x x}+(1 / r)\left(r p_{1 r}\right)_{r} \\
& \quad+\left(k^{2}-n^{2} / r^{2}\right) p_{1}+2 i k m \bar{u}_{0} p_{1 x}=Q,
\end{aligned}
$$

where

$$
\begin{aligned}
Q= & 2 m\left(\bar{u}_{0} \frac{\partial}{\partial x_{1}}+\bar{v}_{0} \frac{\partial}{\partial r}\right)\left(-i k p_{0}+m \bar{u} p_{0 x}\right) \\
& +2 m\left[\left(\bar{v}_{0} / r\right)\left(w_{0} i n-u_{0 x}\right)\right. \\
& \left.+\bar{v}_{0 r}\left(v_{0 r}-u_{0 x}\right)\right]-2 p_{0 x x_{1}} .
\end{aligned}
$$

By virtue of the boundary condition, the particular solution of the first-order coefficient of the pressure can be represented as the sum of two terms:

$$
\begin{aligned}
p_{1}\left(r, x, x_{1}\right)= & C_{l, n} f_{1}\left(r, x_{1}\right) \\
& \times\left[\exp \left(i k \int_{-\infty}^{x} \frac{m \bar{u}_{0}}{1-m^{2} \bar{u}_{0}^{2}} d x\right) g\left(x, x_{1}\right)\right]_{x} \\
& +B\left(x, x_{1}\right) f\left(r, x_{1}\right),
\end{aligned}
$$

where

$$
f\left(r, x_{1}\right)=J_{n}\left(\gamma_{0 l, n} r / \hat{r}\right)+O(\delta)
$$

and $g\left(x, x_{1}\right)$ is given in Eq. (14). The functions $f_{1}$ and $B$ must be determined by direct solution of Eq. (29) and automatically satisfy the first-order boundary condition. Hence, it has no direct bearing on the functional behavior of $C_{l, n}\left(x_{1}\right)$. It is the solution of the first term in Eq. (30) that gives rise to the solvability condition. Therefore, we concentrate our effort on the influence of the first term on the pressure.

By direct substitution of the first term given in Eq. (30), multiplying the result by $r f\left(r, x_{1}\right)$ and integrating the result in the radial direction over the interval $O$ to $\hat{r}$ we find that $C_{l, n}\left(x_{1}\right)$ satisfies the equation

$$
\frac{d}{d x_{1}} C_{l, n}+\beta\left(x_{1}\right) C_{l, n}=O(\delta),
$$


where

$$
\begin{aligned}
& \beta\left(x_{1}\right)=\alpha \frac{d}{d x_{1}} \ln (\hat{r})+\alpha_{1} \frac{d}{d x_{1}} \ln \left(\bar{u}_{0}\right), \\
& \alpha\left(x_{1}\right)=\frac{1-\left(1-m^{2} \bar{u}_{0}^{2}\right)\left(1-2 \Lambda_{l, m}\right)}{2\left(1-m^{2} \bar{u}_{0}^{2} \Lambda_{l, n}\right.}, \\
& \alpha_{1}=\frac{m^{2} \bar{u}_{0}^{2}\left(1-2 \Lambda_{l, n}\right)+2 \Lambda_{l, n}}{2\left(1-m^{2} \bar{u}_{0}^{2}\right) \Lambda_{l, n}},
\end{aligned}
$$

and

$$
\Lambda_{l, n}=\int_{0}^{\hat{r}} \frac{\left[r J_{n}^{2}\left(\gamma_{0 l, n} r / \hat{r}\right)\right] d r}{\left[\hat{r}^{2} J_{n}^{2}\left(\gamma_{0 l, n}\right)\right]} .
$$

Equation (31) is similar to the result given by Uenishi and Meyers for the modulation amplitude of an acoustic disturbance traveling in a two-dimensional waveguide. The only difference is that the first term in the expression for $\beta$ is premultipled by the term $\alpha$. The influence of the vertical component of the mean-flow velocity is manifest in the term $\alpha_{1} d\left[\ln \left(\bar{u}_{0}\right)\right] / d x_{1}$. Therefore, we can say that the vertical velocity serves to modulate the acoustic pressure at lowest order through the variation of the function $C_{l, n}$. Intermodal coupling occurs at $O(\delta)$ through the effect of the mean-flow boundary layer. For small values of the Mach number, $C_{l, n}$ can be approximated as

$$
C_{l, n}=A / \hat{r} \bar{u}_{0}+O\left(m^{2}\right)+O(\delta),
$$

where $A$ is determined by the initial condition placed on the pressure.

\section{RESULTS}

In this section we present some numerical results obtained from the analysis given in the previous sections. Since the validity of the solution given here is predicated on the assumption that the variation of $\hat{k}$ and $\hat{\gamma}$ in Eq. (12) can be effectively modeled by a hypergeometric function having constant arguments, we will first compare the numerical so-

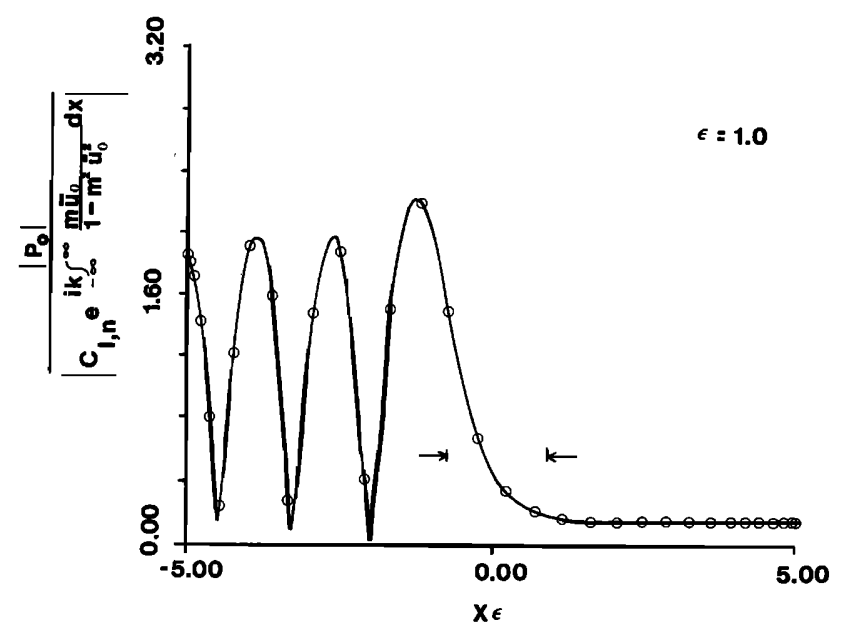

FIG. 2. Magnitude of the $(1,0)$ mode of the acoustic pressure plotted versus axial location for an entrance Mach number of 0.05 . Arrows denote the region of mode cutoff. Circles denote numerical solution of Eq. (12), while the solid line denotes the analytical solution given in Eqs. (15) and (16).

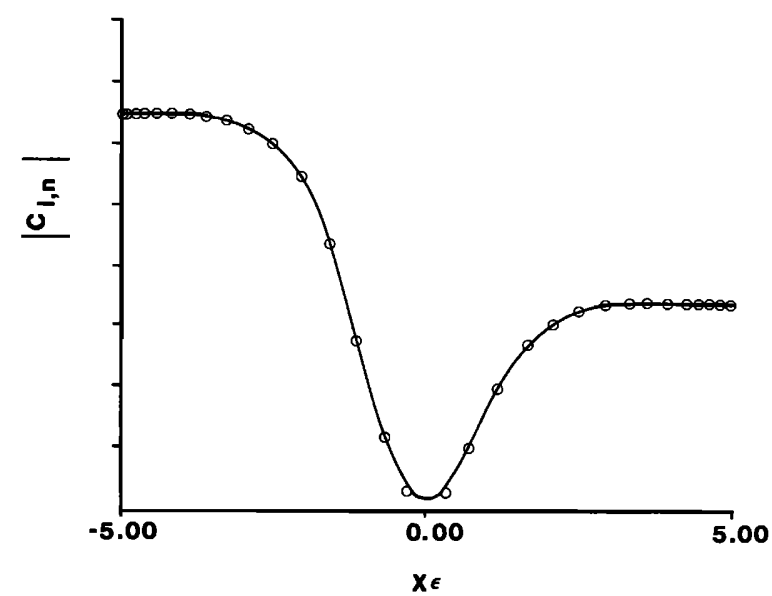

FIG. 3. Magnitude of $C_{l, n}$ plotted versus axial location. Circles denote numerical solution of Eq. (31), while the solid line denotes the analytical solution given in Eq. (32).

lution to those given in Eqs. (15) and (16). In our calculations the mean flow $\bar{u}_{0}$ will be modeled by the expression $1 / \hat{r}^{2}$.

For local Mach numbers ranging between 0.0 and 0.2 we found that the pressure solution given in Eqs. (15) and (16) was in good agreement with the results obtained by direct numerical solution of Eq. (12). Agreement is precise even for values of $\epsilon$ equal to one. This is the result of the model used for the mean flow. In using the aforementioned approximation the error in the pressure is $O\left(\mathrm{~m}^{2}\right)$. Therefore, the hypergeometric function having constant arguments $a, b$, and $c$ accurately represents the pressure solution for small values of Mach number. The result for the pressure for a single geometric configuration will be given here. This configuration corresponds to the case where the entrance radius is 1.0 , the contraction radius is 0.5 , the exit radius is 0.75 , and $\epsilon=1$. In Fig. 2 we have plotted a typical result for the magnitude of the pressure tabulated using Eqs. (15) and (16). In the case plotted, $k$ and $\gamma$ are set equal to $1.00 \pi$ and $0.5861 \pi$, respectively. The value of $\gamma$ chosen corresponds to the $(1,0)$ mode of the duct. The mean flow enters the duct at a Mach number of 0.05 and exits with a Mach number of 0.089 . A maximum of 0.2 occurs in the value of the Mach number at the constriction of the duct. The solid lines represent the pressure solution for the $(1,0)$ mode and the circles represent the values computed numerically. Note that the acoustical mode cuts off in the region of the contraction and cuts on elsewhere. The regions of wave cutoff are denoted by the arrows along the $x$ axis. We see that for values of $x$ which are less than zero we have a standing wave which results from the scattering of the acoustic wave from the constriction. The remaining energy is transmitted to regions of space corresponding to values of $x$ greater than zero.

In Fig. 3, we have plotted the pressure amplitude envelope $C_{l, n}$ for the $(1,0)$ mode under the conditions given above. The numerical results obtained by solving Eq. (31) numerically are denoted in circles while the approximate expression for $C_{l, n}$ is denoted by the solid line. We see that 
the approximate expression is in excellent agreement for small values of Mach number.

\section{v. CONCLUSION}

An analysis of acoustic wave propagation in a slowly varying circular cosh duct carrying an incompressible mean flow has been presented. The solution for the acoustic pressure was obtained in terms of a regular perturbation expansion in $\epsilon$ and a singular expansion in the boundary-layer thickness $\delta$. The singular behavior of the pressure in the mean-flow boundary layer was analyzed using the method of matched asymptotic expansions. It was found that the pressure is affected at $O\left(\delta^{2}\right)$, whereas the eigenvalues of the crossmodes are affected at $O(\delta)$. The refractive effect of the boundary layer is shown to be significant when the meanflow boundary-layer thickness becomes of the order of the crossmode wavelength. The axial variation of the acoustic wavenumber can cause significant difficulties if the wave envelope method is used, especially in the vicinity of turning points. The solution we have given, however, remains uniformly valid through such regions.

\section{ACKNOWLEDGMENTS}

This work was partially supported by the National Science Foundation Grant MEA-8404776 and the Analog Devices Professorship at the University of Lowell. The authors also wish to acknowledge P. Lee for her expert preparation of this manuscript.

'A. H. Nayfeh, B. S. Shaker, and J. E. Kaiser, "Transmission of Sound through Nonuniform Circular Ducts with Compressible Mean Flows," AIAA J. 18, 515-525 (1980).

${ }^{2}$ K. Uenishi and M. K. Myers, "Two-Dimensional Acoustic Field in a Nonuniform Duct Carrying Compressible Flow," AIAA J. 22, 1242-1248 (1984).

${ }^{3}$ L. S. King and K. Karamchetti, "Propagation of Plane Waves in Flow through a Variable Area Duct," AIAA Paper 73-1009 (Oct. 1973).

${ }^{4} R$. J. Alfredson, "The Propagation of Sound in a Circular Duct of Continuously Varying Cross-Sectional Area," J. Sound Vib. 23, 433-442 (1972).

${ }^{5}$ A. Powell, "Theory of Sound Propagation through Ducts Carrying HighSpeed Flows," J. Acoust. Soc. Am. 33, 1640-1646 (1960).

${ }^{6}$ M. K. Myers and S. L. Chuang, "Uniform Asymptotic Approximations for Duct Modes in a Thin Boundary-Layer Flow," AIAA J. 22, 1233-1242 (1984).

'M. Van Dyke, Perturbation Methods in Fluid Mechanics (Parabolic, Stanford, 1964). 